Perspective

\title{
Relax into Yoga for Seniors: An Evidence-Informed Update for Enhancing Yoga Practice Benefits by Reducing Risk in a Uniquely Vulnerable Age Group
}

Carol Krucoff ${ }^{1,}{ }^{*}$, Kimberly Carson ${ }^{2}$, Mitchell Krucoff ${ }^{3}$

1. Duke Integrative Medicine, Duke University, Durham, NC, USA; E-Mail: carol.krucoff@duke.edu

2. Oregon Health \& Science University, Portland, OR, USA; E-Mail: carsonk@ohsu.edu

3. Department of Medicine/Cardiology, Duke University Medical Center, Durham, NC, USA; E-Mail: mitchell.krucoff@duke.edu

* Correspondence: Carol Krucoff; E-Mail: carol.krucoff@duke.edu

Academic Editor: Marieke Van Puymbroeck

Special Issue: $\underline{\text { Yoga in Older Adults }}$

OBM Geriatrics

2021, volume 5, issue 1

doi:10.21926/obm.geriatr.2101150
Received: August 13, 2020

Accepted: December 22, 2020

Published: January 07, 2021

\begin{abstract}
Teaching yoga to seniors is often considered an entry-level job for a yoga instructor since older adults are unlikely to attempt complicated, "advanced" postures. Unfortunately, this ill-informed attitude puts the most vulnerable students with the least-trained instructors who may unknowingly put their students at risk. Even experienced yoga instructors often do not understand the vulnerabilities of older adults, and many commonly taught yoga poses may present risks for seniors. This could explain why participants aged 65 and older have a greater rate of injury from practicing yoga when compared with other age groups. This article discusses the safety principles central to the Integrative Yoga for Seniors program. Offered since 2007 at Duke Integrative Medicine, this professional training for yoga instructors is designed to help yoga teachers enhance the benefits of yoga for their older adult students by learning how to work safely with the increasing number of seniors coming to yoga classes. Drawing on specific Principles of Practice that we developed to integrate the knowledge gained from Western medicine with yogic teachings, we offer our evidence-
\end{abstract}

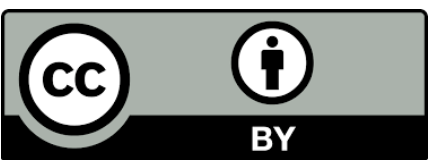

(C) 2021 by the author. This is an open access article distributed under the conditions of the Creative Commons by Attribution License, which permits unrestricted use, distribution, and reproduction in any medium or format, provided the original work is correctly cited. 
informed Relax into Yoga Safety Recommendations to help reduce risk and thus enhance effectiveness when teaching yoga to older adult beginners. We highlight two areas that pose the greatest risk of compromise to older adult students: cardiovascular disease and osteoporosis.

\section{Graphical Abstract}

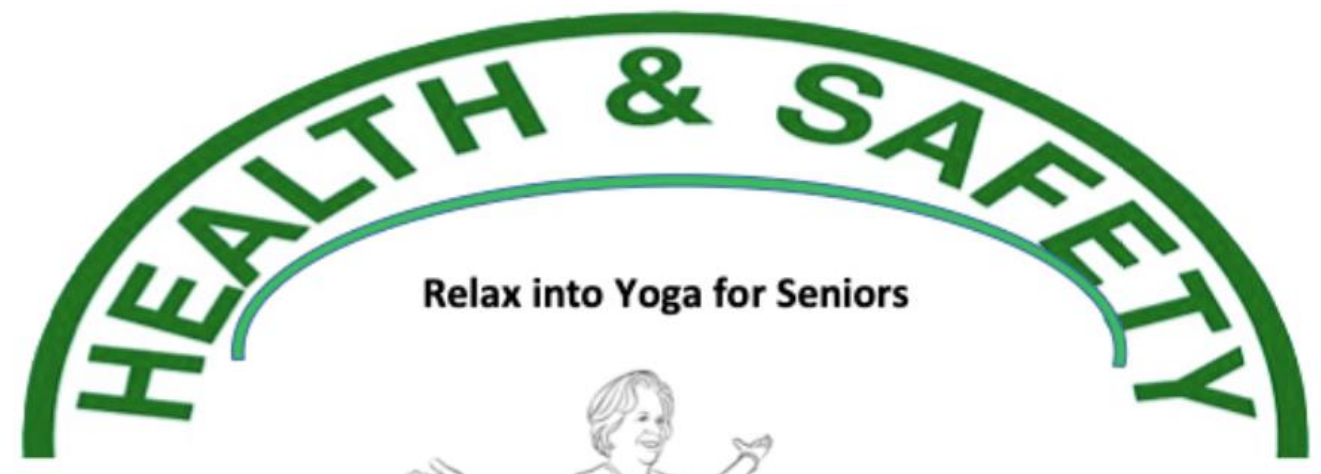

\section{Avoid Extremes}

Keep the Breath Flowing \& Comfortable

Maintain Proper Hydration

Keep Twists in Mid-Range

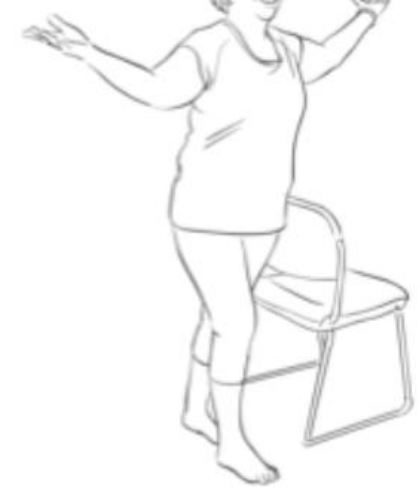

Move Slowly and Mindfully

Avoid Rounding the Spine in Sitting or Standing

Hinge from the Hips

No Straight-Legged Forward Bends

\section{Respect Gravity's Effect on the Bones and Bodily Fluids}

\section{Keywords}

Yoga; seniors; safety; cardiovascular disease; osteoporosis

\section{Introduction}

Yoga may help people age well, according to a growing body of research into its potential benefits for body and mind [1]. These include reducing the risks of age-related "senescent" disorders such as cardiovascular disease and metabolic syndrome, [2] relieving anxiety and depression, [3] easing back pain [4], and enhancing the mental and physical health of sedentary adults with arthritis [5]. One study even described improvements in biological markers of aging in people who practice yoga and meditation, and suggested that these practices "may hold the key to delay aging or aging gracefully" [6]. Supported by this evidence, there is a growing trend for health care providers to "prescribe" yoga as a complementary therapy to help prevent and treat a variety of medical conditions [7]. Thus over the last decade, the number of yoga practitioners in the United States has more than doubled to 36.7 million, with health benefits cited as the main reason why people seek and sustain their practice [8]. 
While media images typically portray yoga enthusiasts as young and fit, in reality, more than a third of Americans who practice yoga are age 50 and older: 17 percent are in their 50s, and 21 percent are age 60 and older [8]. Seniors' strong interest in yoga, during a time when older adults are the fastest growing sector of the population [9], presents a great opportunity and a serious challenge for yoga instructors. As with any therapeutic intervention that offers health benefits, yoga practice also carries potential risks with the most common injuries being sprains and strains [10]. Relatively few serious adverse events have been reported in healthy individuals and are often related to beginners or people with medical preconditions attempting advanced yoga postures such as Headstand (Sirsasana) and Shoulder Stand (Sarvangasana) [11]. This suggests that even older adults who are extremely vital and fit need to keep safety in mind-especially if they are new to yoga practice-as they are more likely to have a medical precondition that may increase their risk of injury. Unfortunately, many yoga instructors are woefully ignorant about the physiology of aging, and some commonly taught yoga poses could be risky for older adults. This may explain why yoga participants aged 65 years and older have a greater rate of injury when compared with other age groups [10].

Of all age groups, seniors are the most complex and varied, with some able to run marathons and others unable to get out of bed. Approximately 85 percent of older adults have at least one chronic health condition, and 60 percent have at least two [12]. In addition to known, diagnosed diseases, seniors are also at a higher risk than younger people for occult disorders that may be aggravated without warning, including hypertension, heart disease and osteoporosis. The first symptom of osteoporosis may be a broken bone, and the first symptom of heart disease may be a heart attack or sudden death.

Adding to the complexity of teaching yoga to older adults is that 90 percent of seniors take at least one prescription drug, and nearly 40 percent take five or more [13]. Several classes of medications commonly taken by seniors-including antihypertensives, antianxiety drugs and antidepressants-are associated with an increased risk of falls. [14]. The use of five or more drugs is associated with a 21 percent increase in the risk of falls [15]. This is of particular concern, since falls are the leading cause of fatal and nonfatal injuries in the US among people age 65 and older, and the most common cause of injuries and hospital admissions for trauma in this age group [16]. Death rates from falls among seniors have increased by 30 percent from 2007 to 2016, according to the Centers for Disease Control and Prevention, which estimates that-if rates continue to risethere will be seven fall deaths every hour by 2030 [17]. These sobering statistics highlight the potential for yoga teachers and yoga therapists to help seniors improve their strength and enhance their balance to reduce risks of falls, with preliminary studies showing a reduction in falls by 48 percent among yoga participants [18].

A further complicating factor is that many older adults who begin yoga are out of shape. More than one in four American adults over age 50 do not engage in regular physical activity-a percentage that jumps to more than one in three for people 75 and older [19]. These data also highlight the potential for physical harm that could occur if yoga instructors do not create a safe environment and class structure geared appropriately to older adults. Finally, seemingly simple expectations in a yoga classroom, such as changing positions from standing to lying on the floor, may be emotionally fraught or even humiliating for seniors unable to lie down and get up again easily. 


\section{The Integrative Yoga for Seniors Program at Duke Integrative Medicine: Perspectives From 13 Years of Professional Trainings}

Medical science offers extensive knowledge about the physiology of aging and the disease process, yet many yoga teachers have limited access to this important information. The Integrative Yoga for Seniors professional training was created to fill the critical need to help yoga instructors work safely and effectively with older adults. Begun in 2007, when Duke University Health System opened its integrative medicine facility (under the original title Therapeutic Yoga for Seniors), this pioneering program started as a three-day workshop and has evolved into its current format as an eight-day, 50-hour professional training, held annually at Duke Integrative Medicine (www.dukeintegrativemedicine.org). As a pre-requisite, participants must be trained yoga teachers, and the curriculum features lectures by Duke University faculty presenters--including physicians, physical therapists, and health psychologists-which focus on health conditions common to seniors, such as heart disease, pulmonary disorders, arthritis, Alzheimer's disease and osteoporosis, and the implications of these disorders to widely-taught yoga postures, breathing techniques, and meditative practices, such as the contemplation of impermanence. The program co-directors are both yoga therapists (and co-authors of this paper) who have decades of experience teaching yoga to older adults in varied medical settings including cardiac rehabilitation, the Gerofit wellness program for older veterans, as well as hospital-based cancer and pain centers. A central part of the training involves experiential sessions designed to help the yoga teachers incorporate the evidence-informed movement considerations recommended by health experts into their yoga practice and teaching. This involves extensive instruction in creative ways to modify poses for enhanced safety and reduced risk for people with varied health conditions. In addition, participants experience movement practices geared to various levels of senior ability-from very fit to chair-dependent-and also learn about special issues in teaching seniors including pain management, spirituality and aging, and end-of-life issues.

In 2010 we published a consolidated overview of our work with safety principles, the CarsonKrucoff Principles of Practice, to promote awareness and provide guidance for yoga teachers who wanted to work safely and effectively with older adults [20]. Informed by our collaboration with Duke medical experts and our extensive experience teaching yoga to seniors, the first of these 12 principles highlights the imperative of safety: "First, Do No Harm. Join with our physician colleagues in making this our primary intention." Other principles offer guidance on specific ways to create a safe environment for older adult yoga students, such as "Invite students to challenge themselves, but never strain," and focus on how a pose feels, rather than how a pose looks [20].

In the ensuing decade, even as awareness of and evidence on the benefits of yoga have spread in the West through our aging population, we have trained more than 1,000 yoga teachers and yoga therapists from across the United States and around the world through our "master training" at Duke, as well as in abridged trainings offered in various locations and online (www.yoga4seniors.com). The majority of our graduates are from the United States, however we have also trained yoga teachers from more than a dozen countries, representing five continents. Most of these teachers offer numerous classes in their communities, making the potential for dissemination of the Relax into Yoga safety principles quite extensive.

We have continued to evolve our evidence-informed approach with emphasis on enhanced benefit from yoga practice through clear and focused awareness of safety concerns for our 
students, which we call "Relax into Yoga." In 2016, we made these practices and principles directly available to motivated seniors themselves through our book, "Relax into Yoga for Seniors" [21]. The first outcomes evidence on the efficacy of the Relax into Yoga program found that it was effective for improving worry, anxiety and sleep among worried older adults. [22]

With safety principles at the heart of our approach, the word "relax" is explicitly used in the title to highlight an important distinction about initiating and sustaining yoga practice that is particularly relevant to seniors. A key characteristic that differentiates this ancient approach to wholeness from modern exercise is the importance of moving to a point of challenge, but not strain. In contrast to Western forms of fitness, which typically encourage striving and pushing to do more, yoga invites each individual to find an appropriate balance between effort and surrender, courage and caution, doing and undoing. Rather than working to "achieve" a particular outcome, the yoga participant is encouraged to ease into a position of stability and comfort-recognizing the yogic wisdom that our true nature is already whole. This kind, mindful, self-compassionate approach is often quite surprising to new students, and we have found that helping people connect to their own inner wisdom can be transformative. We encourage students to start where they are, not where they think they should be. Then we offer the appropriate tools of yogaincluding postures, breathing, and meditation-to help them deepen their awareness and find ease. We use inclusive, nonjudgmental and encouraging language that validates each person's experience, with the intention of creating a safe and welcoming environment that embraces the yogic concept of ahimsa ("nonharming").

Aligned with our practice principle to "create a safe environment" [20], we avoid a teaching technique used in many yoga classes of presenting an ideal form for a particular posture, then suggesting modifications for those unable to "achieve" this pose. Instead, we recommend beginning with whatever variation is accessible to each student. Then we invite those who would like a little more challenge to move into a more-demanding variation, being careful to make it clear that this harder variation is just a suggestion-and not necessary or expected. Especially when working with older adults, it is essential to counter the cultural myth that "more is better" or "no pain no gain," and to honor each person's truth in a way that validates their abilities and choices. Because despite the common misconception that "advancing" in yoga means doing increasingly more difficult postures, our message is that any posture can be "advanced" if it facilitates connection to resilience as balance, flexibility, presence, awareness and love-a central tenet of the yogic teachings: SatChitAnanda (being, consciousness, love). Every physical gesture is a valid opportunity for growth and clarity; wherever someone needs to be is fine. [21]

Longstanding and widening Western experience and the growing body of clinical evidence highlight several mechanistic pathways through which safely practiced yoga could be considered an ideal practice for older adults:

Physical activity. Appropriate yoga stretches and strengthening practices can help seniors meet public health recommendations to do 150 minutes of moderate-intensity aerobic activity a week or to be as physically active as their abilities and conditions allow. [23]

Diaphragmatic (yoga) breathing may reduce stress and positively influence emotional states. [24]

Meditation may relieve chronic pain and be helpful for a variety of conditions including hypertension, irritable bowel syndrome, anxiety and depression, and insomnia. [25] 
Guiding principles of yoga-including loving kindness and self-compassion-may help counter toxic emotions such as anger and hostility, which are associated with increased risk of cardiovascular disease. [26]

Community. Being part of a community of friends practicing together-known in Sanskrit as sangha-may help counter isolation and loneliness, which can be common among older adults and may be detrimental to cardiovascular health. [27]

Safety-oriented Relax into Yoga is an approach that can be used to optimize yoga practice for an individual facing virtually any health challenge. Examples follow for two common ailments that pose the greatest risk of compromise to older adult students: cardiovascular disease and osteoporosis.

\section{Cardiovascular Disorders}

Age is one of the most significant risk factors both for the development of and for morbidity and mortality related to all cardiovascular ailments [28]. This includes coronary artery disease (CAD), which is the number one cause of death and disability for both men and women in the United States as well as numerous other chronic and potentially life-threatening cardiovascular conditions such as hypertension, congestive heart failure, arrhythmias, valvular disorders and stroke. Senescent changes include a stiffening of elasticity in the blood vessels, which can impact blood pressure, and on the heart muscle itself, which can lead to symptoms of heart failure. Over decades of life, wear and tear on the blood vessels can lead to aneurysms and clots and on the heart valves can lead to narrowing or leaking [20].

A result of these common cardiovascular changes is that gravitational shifts common in yoga practice-such as moving from lying down to standing up--may have a marked effect on the older adult body. For example, moving to an upright position from supine to sitting, or moving from sitting to standing may lead to dizziness and increased risk of falling, a condition called orthostatic hypotension [29]. This is why, in our Safety Recommendations for Cardiovascular Concerns (see Table 1), we advise moving slowly and mindfully when changing positions and having a chair or other support nearby for stability. Another example of gravity's effect on the cardiovascular system is that, for some people with heart issues, having the legs at or above the level of the heart may lead to breathlessness. In this case, the posture may be modified by lowering the legs or raising the chest. Another gravity-related cardiovascular concern is that bringing the head below the heart may result in discomfort and/or headache, and possibly increase the risk of stroke. Our safety recommendations include avoiding head-below-the-heart positions for older adults with cardiovascular risk factors and those new to the practice (see Table 1). Respiration can also influence cardiovascular function-for example holding the breath can cause significant changes in heart rate and arterial blood pressure [30]. Yoga breathing techniques that involve sustained rapid or forceful breathing, or breath holding, may also lead to discomfort, dizziness, risk of falling and even loss of consciousness. We recommend avoiding extreme breathing practices, teaching proper breathing mechanics, and inviting students to keep their breath flowing and comfortable (see Table 1). 
Table 1 Relax into Yoga Safety Recommendations for Cardiovascular Concerns.

- Move slowly and mindfully when changing positions-i.e. from lying down to standing. Take your time and have support (a chair or wall) nearby.

- Avoid breath holding. Holding the breath can affect blood pressure. In particular, avoid holding the breath while straining-a practice known as the Valsalva maneuver-which can occur in daily activities, such as lifting something heavy or straining while going to the bathroom.

- Avoid any kind of forceful breathing. Any practice that results in a feeling of struggling for breath should be avoided. Breathing practices that encourage a gentle suspension of the breath at the end of the exhalation may be fine, but participants are encouraged to check with their physician if they have any concerns.

- Keep the breath flowing and comfortable. Teaching proper breathing mechanics can be extremely valuable, particularly in a culture where elevated stress levels frequently result in shallow and inefficient breathing. In addition, helping people learn breath awareness-the simple, yet powerful skill of paying attention to the natural breath-can be extremely important to physical and mental health. The empowering recognition that breathing can be a reflection of what's going on in the body, heart, and mind offers older adults an important skill that they can take off the yoga mat and into daily life.

- Avoid extremes. This includes extreme movements, extreme exertion, extreme breathing practices and extreme temperatures, all of which can strain the cardiovascular system.

- Avoid head-below-the-heart positions. While someone who has been practicing yoga for decades may be fine doing head-down inversions, we advise against these postures for older adults with cardiovascular risk factors and those new to the practice.

- Avoid dropping the head back. Extreme cervical extension may cause dizziness and, potentially, a stroke.

- Maintain proper hydration. Encourage students to drink some water before practicing as dehydration could result in fatigue, dizziness and/or fainting.

- Recognize that relaxation is important for cardiovascular health. Never underestimate the benefits of practicing being still and quieting body and mind.

*Adapted from Relax into Yoga for Seniors, CKimberly Carson and Carol Krucoff.

\section{Osteoporosis}

The word "osteoporosis" means "porous bones" and is a disorder of impaired bone strength that results in skeletal fragility and increased fracture risk [31]. This bone loss generally occurs in women after menopause and in both men and women with aging [32]. Aging increases the likelihood of developing osteoporosis, with the disorder affecting about 15 percent of women in their 50s and about half of all women in their eighties. [33]. Ethnicity and race, like sex, influences the epidemiology of fractures, with highest fracture rates in white women [34]. Four out of every 10 white women age 50 or older in the United State will experience a hip, spine or wrist fracture during the remainder of their lives [35]. Although bone mineral density is higher in African Americans, these women are more likely to die after hip fracture, have longer hospital stays, and are less likely to be ambulatory at discharge [34]. Fractures can have devastating consequences, 
with hip fractures in the elderly associated with increased mortality [36]. While postmenopausal women are more likely to develop the condition than men, osteoporosis affects men as well, with men over fifty more likely to break a bone from osteoporosis than to get prostate cancer [37]. Having a small, thin body frame increases the risk of osteoporosis, as do certain lifestyle factors and medication use. These include smoking, excessive alcohol use, and long-term use of glucocorticoids, proton pump inhibitors, selective serotonin receptor inhibitors, and some cancer chemotherapeutic drugs [38].

Since osteoporosis has no obvious symptoms, it's possible to have compromised bone density and not know it. In fact, some people learn they have osteoporosis only after they break a bone. Osteopenia, which is the name given to low bone density that is not low enough to be called osteoporosis, also puts people at higher risk of fracture. Surprisingly, it's not uncommon for people to suffer a vertebral fracture and be unaware that this has occurred, since an estimated two-thirds of vertebral fractures do not come to clinical attention [39]. This suggests that these fractures may occur either without pain or pain that is misidentified-as a pulled muscle, for example.

Physical activity can enhance bone health, particularly weight-bearing exercises like walking and resistance exercises such as lifting weights [40]. Many yoga practices and postures involve weight-bearing and resistance and can be extremely beneficial in building and/or maintaining strength and balance and in helping to restore function and relieve pain for people with compromised bone density. Weight-bearing postures such as Warrior Two, (Virabhadrasana II) and Chair Pose, (Utkatasana), may help strengthen muscles and bones, as can poses that move the body against resistance, such as a Yoga Push-Up, which is a less-challenging and more accessible variation of the yoga pose, Chaturanga Dandasana, done with the knees on the ground. However, some commonly-taught yoga poses should be appropriately modified because they have the potential to do significant harm. In particular, spinal flexion (forward bending) and rotation of the spine (twisting) can place large and compromising loads on the front of vertebral bodies [41] and are often implicated in vertebral fracture [42]. For this reason, the National Osteoporosis Foundation (NOF) cautions that "many exercises and activities such as yoga, Pilates, tennis and golf may need to be avoided or adjusted because they often involve twisting and bending motions." [43] The NOF recommends that people diagnosed with osteoporosis:

*Avoid bending forward from the waist

*Avoid twisting the spine to a point of strain

*Avoid having a slumped, head-forward posture

*Avoid sit-ups, abdominal crunches and toe touches [43].

It's essential to consider these precautions in adapting yoga postures appropriately, as some of these contraindicated movements resemble commonly-taught yoga poses. For example, a "toe touch" is similar to a standing, straight-legged forward bend-known in Sanskrit as the yoga pose Uttanasana. Doing a seated version of yoga's "Cat-Cow," (Bidalasana-Bitilasana) pose involves moving into a slumped, head-forward posture. Recognizing the significant risk presented by certain postures, along with the fitness level and abilities of most people who start yoga in their senior years, the Relax into Yoga canon of postures does not include any straight-legged forward bends or "end-range" twists-a move often used in yoga postures where the spine is rotated as much as possible, then leveraged further (for example by pressing a hand into the thigh) to invite even more twist (see Table 2). Our Relax into Yoga for Seniors Safety Recommendations for 
Osteoporosis and Compromised Bone Density emphasizes the importance of teaching students good body mechanics. This includes hinging at the hips (not at the waist), bending the knees, and keeping the spine in a neutral alignment when bending forward (see Table 2). All twists are to be kept in mid-range with a fluid quality of motion.

Table 2 Relax into Yoga Safety Recommendations for Osteoporosis and Compromised Bone Density.

- Learn how to come into a "neutral spine" position. In sitting, sit on the "sit bones" and lengthen up from the top of the head. In standing, keep the pelvis in neutral and lengthen up from the top of the head. Keep the shoulders relaxed and imagine a light at the center of the chest shining forward-not down.

- Avoid rounding the spine in sitting or standing. The safest plane of action for spinal flexion is lying down-for example, supine knees-to-chest pose or side-lying gentle curl.

- Hinge from the hips, not the waist. Do this with any standing or seated postures that involve bending forward. Remember to tilt the pelvis forward, keep the spine long and avoid rounding the back. In standing movements that bend the body forward, hinging from the hips and bending the knees can help maintain proper spinal alignment.

- Avoid twisting the spine forcefully and/or to the end of its range of rotation. It's not uncommon for some yoga poses to twist the spine as far as it will go, then add leverage by pressing a hand or arm against something to twist further. We advise against this "end-range" rotation.

- Keep twists in mid-range, using a smooth quality of motion. While extreme twists are inadvisable, it's important to maintain the spine's rotational mobility as this is an important, functional movement. Be sure to lengthen up from the top of the head before twisting and use a smooth quality of motion to rotate the spine, going just into a mid-range-similar to the movement necessary to back a car out of a parking spot.

- Avoid placing body weight on the neck and/or shoulders. This includes avoiding postures such as Headstand (Sirsasana), Shoulder Stand (Sarvangasana) and Plow (Halasana).

- Have a support nearby. Practice standing poses near a chair or a wall to help maintain balance if necessary.

- Keep the head on the floor when lying supine. Avoid lifting the head and shoulders into a "crunch" or sit-up.

- Move with a smooth, relaxed quality of motion. Avoid jerky, forceful movement.

- Consider lying down or standing rather than sitting to "take a load off" your spine.

Recognize that lying down places the least stress on the spine and may be the safest plane of action for people with compromised bone density to practice many poses. Someone who can't get down onto the floor can do lying-down postures in bed. Standing with good posture is the next safest way to practice poses. Sitting places the most stress on the spine, which means it's particularly important to be mindful of these recommendations when doing seated poses.

*Adapted from Relax into Yoga for Seniors, @Kimberly Carson and Carol Krucoff. 
Seated postures can present a particular challenge for people with compromised bone density because sitting puts more strain on the spine than does standing or lying down, increasing the risk of fracture. In addition, since most people in Western cultures are not accustomed to sitting on the floor, they are typically unable to do so while keeping their spine in a neutral position. Even with the aid of numerous props, floor sitting typically leads to a slumped, collapsed spine, which may invite vertebral fracture. For this reason, Relax into Yoga teaches all seated postures in a chair. Since most people spend much of their days sitting in chairs, yoga practice can be an ideal environment to teach older adults how to sit with good alignment as well as how to stand with good posture and move with healthy body mechanics. Because despite the recommendation that people diagnosed with osteoporosis receive education about proper posture and fracture prevention [20], it is our experience that few people with osteoporosis have learned these essential skills. These important alignment lessons learned on the yoga mat can help people protect their bones in daily life.

\section{Relax into Yoga Posture Variations}

Learning how to skillfully translate movement considerations that are informed by Western medical science into safe, effective, and enjoyable variations of classic yoga postures is a central focus of our Integrative Yoga for Seniors professional trainings. Our approach to creating evidence-informed posture variations involves identifying three things:

1. What is the intention of the pose? Do we practice this posture to strengthen the core, enhance the flexibility of the hips, stretch the legs, or maybe all three? Define the purpose for why you are teaching/doing a particular posture.

2. What are the risks of the pose? Could this posture put pressure on the knees, cause someone to fall, or strain their wrists? Determine the potential problem points in the posture.

3. What are the specific limitations of the student? Did this person have a recent hip replacement? Is he on medication that makes him dizzy? Is she in persistent pain? Be clear about the issues of a particular student to decide how to meet some or all of the intentions of a pose while minimizing the risks.

As teachers begin to consider the "canon" of yoga postures from this perspective it may require some "deconstruction"-that is viewing each posture in terms of specific benefits and risks. Here are three examples of the Relax into Yoga approach to creating accessible posture variations that meet the intention of the pose, while reducing the risks this movement might present to an older adult.

\subsection{Puppy Dog rather than Downward-Facing Dog (Adho Mukha Svanasana)}

A central intention of Downward-facing Dog Pose is to stretch the back body. However, this posture can be risky for seniors with compromised bone density, heart issues and/or arthritis as it can bring people into loaded spinal flexion, places the head below the heart, and may put excessive demands on the wrists and shoulders. "Puppy Dog" places the palms on a wall, rather than the floor, and invites a similar stretch of the back body, without these compromising factors. 

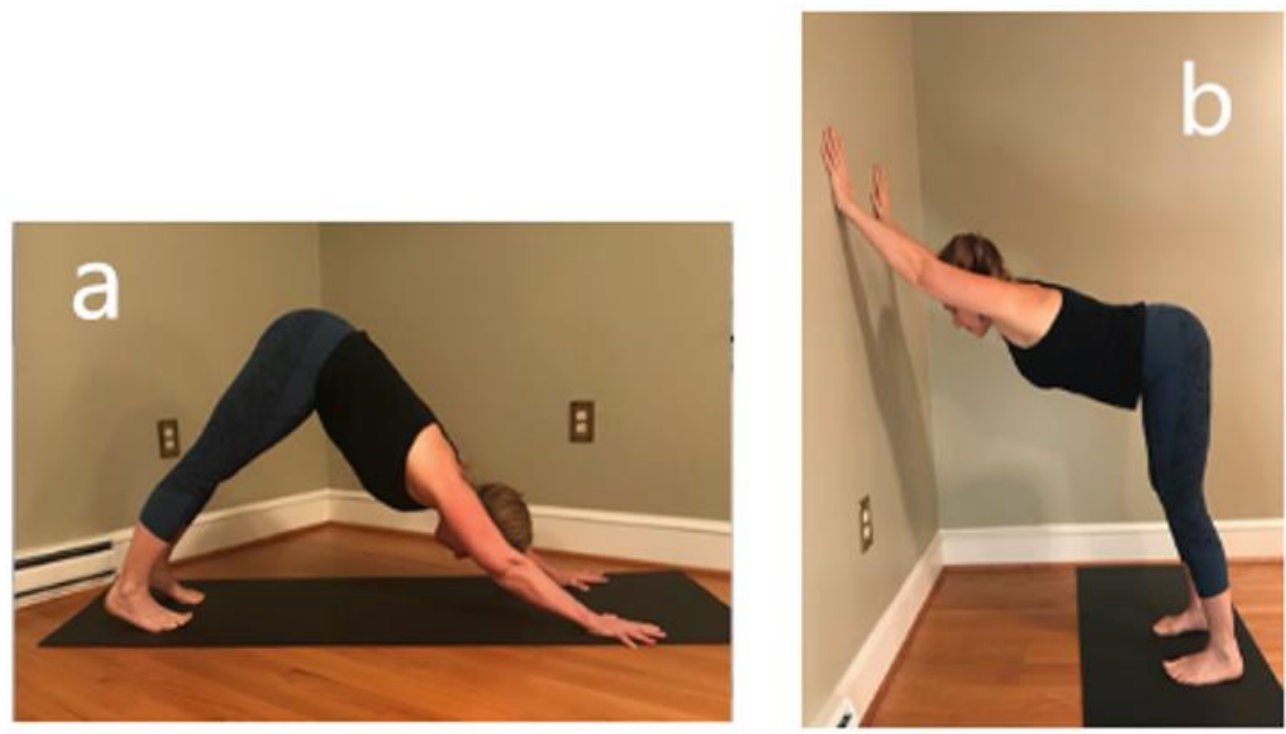

Figure 1 Downward-facing Dog and Relax into Yoga "Puppy Dog".

\subsection{Tree Pose (Vrikshasana) Variations}

Main intentions of Tree Pose (Vrikshasana) are to boost balance and build core strength. Yet bringing one foot to the top of the opposite thigh in the classic expression of this balance pose is likely inaccessible to most older adults and poses a fall risk. And for older adults with arthritis in the knees and/or hips, placing all the body weight on one leg may be quite painful. Relax into Yoga variations still challenge balance and strengthen the core, while reducing fall risk with the use of a support. These variations--in which some of the body's weight is taken off the standing leg, for example, by touching the opposite toe to the ground--are also more accessible for people with tender knees and hips.
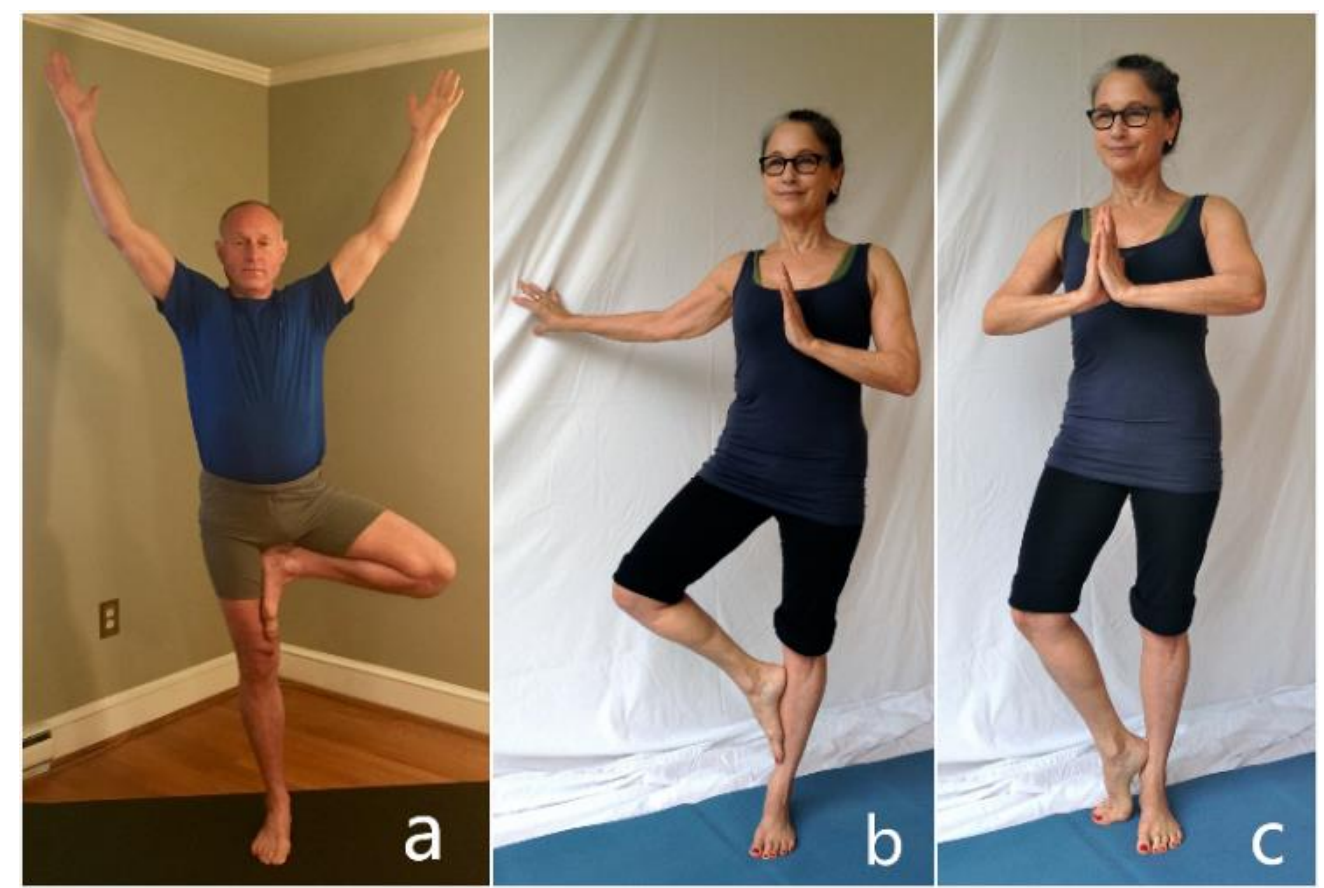

Figure 2 Tree Pose, Relax into Yoga Tree Pose Variations. 


\subsection{Triangle Pose (Trikonasana) Variations}

Opening the chest and stretching the side body, while strengthening the legs, are main intentions of Triangle Pose. However attempting to bring the hand down to the shin, ankle or floor can pose risks of bringing the spine into loaded flexion and potentially compromising rotation. Using a chair to support the Triangle offers similar stretching and strengthening benefits, while reducing these risks.
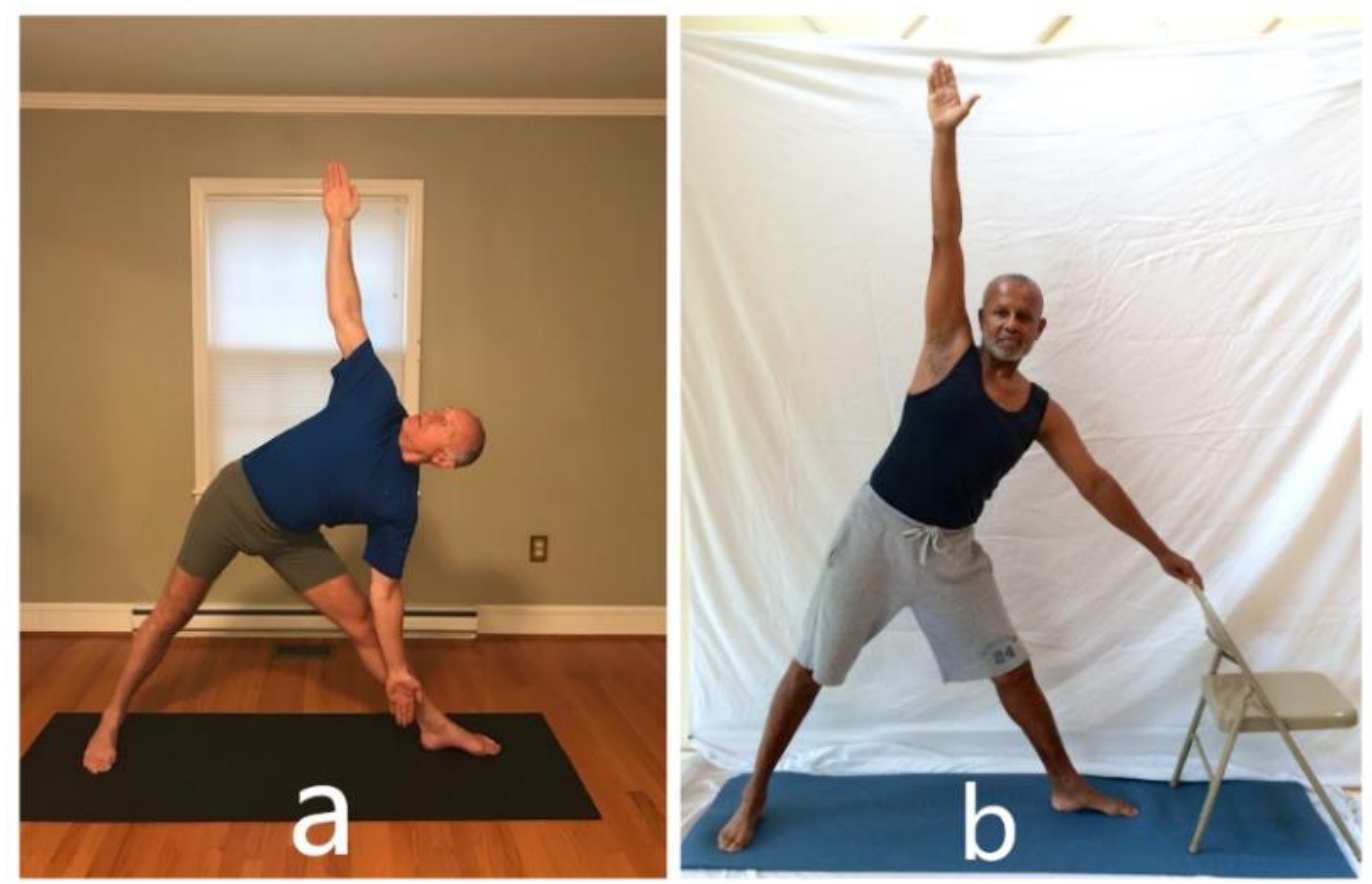

Figure 3 Triangle Pose and Relax into Yoga Triangle Variation.

\section{Relax into Yoga Recommendations for Balancing Risks and Rewards}

Relax Into Yoga for Seniors has developed and continues to evolve safety-oriented recommendations designed to enhance benefit by reducing risk for older adults beginning a yoga practice-recognizing that the majority of Western seniors are likely to be relatively deconditioned and facing numerous health challenges. In the decade since publishing the Carson-Krucoff Principles of Practice [20] as a skillful framework for teaching yoga to seniors, the Relax into Yoga principles, postures and practices have evolved through a combination of student-centered feedback and the advance of scientific reports related to safety and movement considerations recommended for people with specific disorders.

Central to Relax Into Yoga is the emphasis on postures that facilitate functional movementssuch as sitting and standing with good posture, getting up and down from a chair with ease, enhancing and maintaining good balance, and being able to back a car out of a parking space. Unlike younger practitioners who often come to yoga to look better, most older adults practice yoga to feel and function better. Seniors' motivation for yoga practice is often to relieve pain and stiffness, ease stress, boost mood and enhance their ability to remain independent---driving a car, getting up and down from the floor to play with grandchildren, being able to maintain their house and yard. 
It is also important to recognize that individual seniors may be exceptional-they may be quite fit and able and perhaps have practiced yoga for decades. If a 70-year-old has been standing on their head every day since childhood, that practice may be fine for that person. However the reality is that, even fairly active seniors with athletic backgrounds who are just starting yoga may have conditions that require special consideration. By virtue of age alone, older adults are at greater risk for both known and occult health issues, which suggests that even though someone can do a particular posture, it may still be risky-and inadvisable--for them to do it. The Relax into Yoga approach can be as vigorous as appropriate and still follow these risk-reduction principles. Many challenging yoga poses meet the Relax into Yoga Safety Recommendations and may be available to older adults who have the physical ability and experience, including Plank (Kumbhakasana) and Side Plank (Vasisthasana), Eagle Pose (Garudasansa), Warrior Three (Virabhadrasana III), Half Moon Pose (Ardha Chandrasana), and Chair Pose (Utkatasana).

In the Western world, awareness of and evidence on the benefits of yoga practice have greatly expanded over the past decade and is likely to continue to do so as the National Library of Medicine now lists more than 800 studies on its website (ClinicalTrials.gov) examining the therapeutic benefits of yoga for varied conditions including Chronic Obstructive Pulmonary Disease, Parkinson's Disease, heart failure and osteoarthritis. As the science continues to advance, we consider it essential that yoga instructors who include or concentrate on seniors magnify the mind-body-spirit potential of their teaching by ensuring the safety of their students through appropriate teacher trainings and practitioner certification.

It's also important to recognize that, as yoga has become increasingly popular, the word "yoga" is typically used to refer to the physical aspect of the much fuller discipline. But posture-known as asana-is just one tool in yoga's extensive toolkit, which also includes breathing, meditation and guiding principles. Posture practice offers a unique opportunity to assist in the exploration of what it means to be human, to become aware of the subtle energies within, and to connect with our own deep-down goodness. In the ancient teaching of yoga, love is considered a defining virtue of the human experience. Relax into Yoga centers on cultivating a loving approach to every aspect of practice, including moving in and out of postures, relationships to thoughts and feelings and receptivity to energies, sensations and spirit. For many seniors, safe and successful engagement of this dimension of yoga practice may in fact produce the greatest benefits of all.

\section{Author Contributions}

Carol Krucoff conceived and developed the Relax into Yoga for Seniors Program, wrote the first and final draft of this manuscript and serves as the primary author. Kimberly Carson conceived and developed the Relax into Yoga for Seniors Program, and provided intellectual and editorial contribution to this manuscript. Mitchell Krucoff provided intellectual and editorial contribution to this manuscript.

\section{Competing Interests}

The authors have declared that no competing interests exist. 


\section{References}

1. Mooventhan A, Nivethitha L. Evidence based effects of yoga practice on various health related problems of elderly people: A review. J Bodyw Mov Ther. 2017; 21: 1028-1032.

2. Chu P, Gotink RA, Yeh GY, Goldie SJ, Hunink MM. The effectiveness of yoga in modifying risk factors for cardiovascular disease and metabolic syndrome: A systematic review and metaanalysis of randomized controlled trials. Eur J Prev Cardiol. 2016; 23: 291-307.

3. Vollbehr NK, Bartels-Velthuis AA, Nauta MH, Castelein S, Steenhuis LA, Hoenders HR, et al. Hatha yoga for acute, chronic and/or treatment-resistant mood and anxiety disorders: A systematic review and meta-analysis. PLoS One. 2018; 13: e0204925.

4. Saper RB, Lemaster C, Delitto A, Sherman KJ, Herman PM, Sadikova E, et al. Yoga, physical therapy, or education for chronic low back pain: A randomized noninferiority trial. Ann Intern Med. 2017; 167: 85-94.

5. Moonaz SH, Bingham 3rd CO, Wissow L, Bartlett SJ. Yoga in sedentary adults with arthritis: Effects of a randomized controlled pragmatic trial. J Rheumatol. 2015; 42: 1194-1202.

6. Tolahunase M, Sagar R, Dada R. Impact of yoga and meditation on cellular aging in apparently healthy individuals: A prospective, open-label single-arm exploratory study. Oxid Med Cell Longev. 2017; 2017: 7928981.

7. Stephens I. Medical yoga therapy. Children. 2017; 4: 12.

8. 2016 Yoga in America study [Internet]. New York: Ipsos Public Affairs; 2016. Available from: https://static-yogajournal.s3.amazonaws.com/wp-content/uploads/2016-Yoga-in-AmericaStudy-Comprehensive-RESULTS.pdf.

9. 65 and older population grows rapidly as baby boomers age. Available from: https://www.census.gov/newsroom/press-releases/2020/65-older-population grows.html.

10. Swain TA, McGwin G. Yoga-related injuries in the United States from 2001 to 2014. Orthop J Sports Med. 2016; 4: 2325967116671703.

11. Cramer H, Quinker D, Schumann D, Wardle J, Dobos G, Lauche R. Adverse effects of yoga: A national cross-sectional survey. BMC Complement Altern Med. 2019; 19: 190.

12. Supporting older patients with chronic conditions. Bethesda: National Institute on Aging; 2017. Available from: https://www.nia.nih.gov/health/supporting-older-patients-chronic-conditions.

13. Kantor ED, Rehm CD, Haas JS, Chan AT, Giovannucci EL. Trends in prescription drug use among adults in the United States from 1999-2012. JAMA. 2015; 314: 1818-1831.

14. De Jong MR, Van der Elst M, Hartholt KA. Drug-related falls in older patients: Implicated drugs, consequences, and possible prevention strategies. Ther Adv Drug Saf. 2013; 4: 147-154.

15. Dhalwani NN, Fahami R, Sathanapally H, Seidu S, Davies MJ, Khunti K. Association between polypharmacy and falls in older adults: A longitudinal study from England. BMJ Open 2017; 7: e016358.

16. Bergen G, Stevens MR, Burns ER. Falls and fall injuries among adults aged $>65$ Years-United States, 2014. MMWR Morb Mortal Wkly Rep. 2016; 65: 993-998.

17. Important facts about falls. Atlanta: Centers for Disease Control and Prevention; 2017. Available from: https://www.cdc.gov/homeandrecreationalsafety/falls/adultfalls.html.

18. Hamrick I, Mross P, Christopher N, Smith PD. Yoga's effect on falls in rural, older adults. Complement Ther Med. 2017; 35: 57-63. 
19. Physical activity. Atlanta: Centers for Disease Control and Prevention; 2016. Available from: https://www.cdc.gov/media/releases/2016/p0915-physical-activity.html.

20. Krucoff C, Carson K, Peterson M, Shipp K, Krucoff M. Teaching yoga to seniors: Essential considerations to enhance safety and reduce risk in a uniquely vulnerable age group. $J$ Altern Complement Med. 2010; 16: 899-905.

21. Carson K, Krucoff C. Relax into yoga for seniors: A six-week program for strength, balance, flexibility and pain relief. Oakland: New Harbinger Publications; 2016.

22. Brenes GA, Divers J, Miller ME, Anderson A, Hargis G, Danhauer SC. Comparison of cognitivebehavioral therapy and yoga for the treatment of late-life worry: A randomized preference trial. Depress Anxiety. 2020; 37: 1194-1207.

23. Physical activity guidelines for americans. Washington: Department of Health \& Human Services. Available from: https://health.gov/our-work/physical-activity.

24. Ma X, Yue ZQ, Gong ZQ, Zhang H, Duan NY, Shi YT, et al. The effect of diaphragmatic breathing on attention, negative affect and stress in healthy adults. Front Psychol. 2017; 8: 874.

25. Meditation: In depth. Maryland: National Center for Complementary and Integrative Health; 2016. Available from: https://www.nccih.nih.gov/health/meditation-in-depth.

26. Suls J. Anger and the heart: Perspectives on cardiac risk, mechanisms and interventions. Prog Cardiovasc Dis. 2013; 55: 538-547.

27. Xia N, Li H. Loneliness, social isolation, and cardiovascular health. Antioxid Redox Signal. 2018; 28: 837-851.

28. Rodgers JL, Jones J, Bolleddu SI, Vanthenapalli S, Rodgers LE, Shah K, et al. Cardiovascular risks associated with gender and aging. J Cardiovasc Dev Dis. 2019; 6: 19.

29. Mol A, Hoang PT, Sharmin S, Reijnierse EM, van Wezel RJ, Meskers CG, et al. Orthostatic hypotension and falls in older adults: A systematic review and meta-analysis. J Am Med Dir Assoc. 2019; 20: 589-597.

30. Grunovas A, Trinkunas E, Buliuolis A, Venskaityte E, Poderys J, Poderiene K. Cardiovascular response to breath-holding explained by changes of the indices and their dynamic interactions. Biol Syst Open Access 2016; 5: 152.

31. NIH Consensus Development Panel on Osteoporosis Prevention, Diagnosis, and Therapy. Osteoporosis prevention, diagnosis and therapy. JAMA. 2001; 285: 785-795.

32. Clarke BL, Khosla S. Physiology of bone loss. Radiol Cli. 2010; 48: 483-495.

33. Praemer A, Fumer S, Rice DP. Musculoskeletal conditions in the United States. Am Acad Orthop Surg. 1999: 182.

34. Cauley JA. Defining ethnic and racial differences in osteoporosis and fragility fractures. Clin Orthop Relat Res. 2011; 469: 1891-1899.

35. Bone health and osteoporosis: A report of the surgeon general. Final report. Rockville: US. Department of Health and Human Services. 2004.

36. Panula J, Pihlajamäki H, Mattila VM, Jaatinen P, Vahlberg T, Aarnio P, et al. Mortality and cause of death in hip fracture patients aged 65 or older: A population-based study. BMC Musculoskelet Disord. 2011; 12: 1-6.

37. Just for men [Internet]. Arlington: National Osteoporosis Foundation. Available from: https://www.nof.org/preventing-fractures/general-facts/just-for-men/. 
38. Panday K, Gona A, Humphrey MB. Medication-induced osteoporosis: Screening and treatment strategies. Ther Adv Musculoskelet Dis. 2014; 6: 185-202.

39. Fechtenbaum JC, Cropet S, Kolta B, Verdoncq P, Orcel P, Roux C. Reporting of vertebral fractures on spine X-rays. Osteoporos Int. 2005; 16: 1823-1826.

40. Exercise for Your Bone Health. Bethesda: National Institute of Arthritis and Musculoskeletal and Skin Diseases. Available from: https://www.bones.nih.gov/health-info/bone/bonehealth/exercise/exercise-your-bone-health.

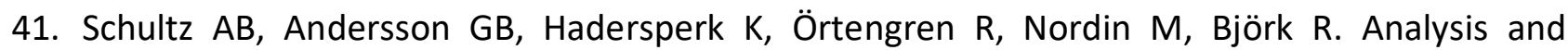
measurement of lumbar trunk loads in tasks involving bends and twists. J Biomech. 1982; 15: 669-675.

42. Melton LJ, Chao EY, lane J. Biomechanical aspects of fractures. In: Riggs BJ, Melton Lj, eds. Osteoporosis: Etiology, diagnosis, and management. New York: Raven Press; 1998. p.119p.122.

43. Proper Body Alignment. Arlington: National Osteoporosis Foundation. Available from: https://www.nof.org/patients/treatment/exercisesafe-movement/proper-body-alignment/.

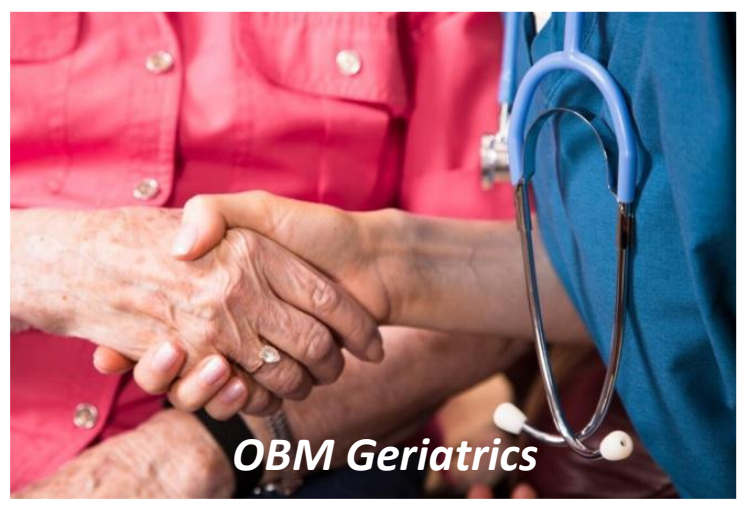

Enjoy OBM Geriatrics by:

1. Submitting a manuscript

2. Joining in volunteer reviewer bank

3. Joining Editorial Board

4. Guest editing a special issue

For more details, please visit: http://www.lidsen.com/journals/geriatrics 